\title{
Índice de vulnerabilidad de adultos mayores en Medellín, Barranquilla y Pasto
}

\author{
Doris Cardona ${ }^{1}$, Ángela Segura², Alejandra Segura ${ }^{3}$, Diana Muñoz ${ }^{1}$, Daniel Jaramillo', Douglas \\ Lizcano', Maite Catalina Agudelo', Catalina Arango' ${ }^{1}$, Santiago Morales ${ }^{3}$ \\ 1 Grupo de Investigación en Epidemiología y Bioestadística, Universidad CES, Medellín, Colombia \\ 2 Grupo de Investigación Observatorio de la Salud Pública, Universidad CES, Medellín, Colombia \\ 3 Grupo de Investigación en Psicología, Salud y Sociedad, Universidad CES, Medellín, Colombia
}

Introducción. La vulnerabilidad puede entenderse como la carencia de recursos materiales e inmateriales que impide el aprovechamiento de oportunidades en distintos aspectos de la vida. Estos recursos de bienestar evitan el deterioro de la calidad de vida.

Objetivo. Construir un índice de vulnerabilidad con las características de los capitales físico, humano, social y funcional de los adultos mayores de tres ciudades de Colombia en el 2016, y determinar los factores asociados con esta condición.

Materiales y métodos. Se hizo un estudio transversal con información primaria mediante 1.514 encuestas a personas de 60 años o más de Medellín, Barranquilla y Pasto. En la construcción del índice se usó el análisis factorial con los métodos de componentes principales y de rotación ortogonal varimax. Resultados. Las condiciones que generaban vulnerabilidad se relacionaron principalmente con el capital humano (calidad de vida, salud mental y hábitos); los demás capitales aportaron un solo componente, así: capital físico (ocupación), capital social (acompañamiento) y capital funcional (independencia funcional). La vulnerabilidad fue mayor en los residentes de Pasto. Los factores asociados con la vulnerabilidad fueron la ciudad de residencia, el sexo, el nivel educativo y el rol en el hogar.

Conclusión. En el $58,55 \%$ de las personas mayores, la vulnerabilidad se explicó por el uso del tiempo, la independencia funcional y el bienestar subjetivo. Estos hallazgos aportan elementos para el mejoramiento de la calidad de vida, principalmente en cuanto a la capacidad funcional para mantener la independencia, estar ocupados y fortalecer la salud mental.

Palabras clave: anciano; anciano frágil; análisis de vulnerabilidad; salud mental; ocupaciones; envejecimiento.

doi: https://doi.org/10.7705/biomedica.v38i0.3846

\section{Index of vulnerability of elderly people in Medellín, Barranquilla, and Pasto}

Introduction: Vulnerability can be defined as a lack of material and immaterial resources, which prevents the use of opportunities that may advance one's self-interest. The presence of these welfare resources prevents reductions in the quality of life.

Objective: The objective of this study was to build an index of vulnerability using characteristics of the physical, human, social and functional capital of adults in three cities of Colombia in the year 2016 and to determine the factors that contribute most to vulnerability.

Materials and methods: We conducted a transversal study with primary information sources applying 1,514 surveys among people aged 60 years and over in Medellín, Barranquilla, and Pasto. For the construction of the vulnerability index, we used factor analysis with varimax rotation and the principal component method.

Results: The conditions that lead to a person's vulnerability were related mainly to human capital (quality of life, mental health and habits). The other types of capital that contributed to vulnerability were physical capital (occupation), social capital (accompaniment), and functional capital (functional independence). The highest vulnerability was registered among the residents of Pasto. The factors associated with vulnerability were the city of residence, the sex, the educational level and the role of the person in the home.

Conclusion: In $58.55 \%$ of elderly people vulnerability was explained by the use of time, the functional independence and the subjective well-being. These findings contribute to the improvement of the

\section{Contribución de los autores:}

Todos los autores participamos en la concepción, la planificación, el diseño, la interpretación y el análisis de los datos, y en la redacción del manuscrito. 
quality of life, mainly those related to maintaining functional independence as long as possible, being occupied, improving mental health, and avoiding the risks of depression, anxiety, cognitive deterioration and deleterious habits.

Key words. Aged; frail elderly; vulnerability analysis; mental health; occupations; aging. doi: https://doi.org/10.7705/biomedica.v38i0.3846

Los estudios sobre la vulnerabilidad han cobrado gran importancia en los últimos años. Según varios autores, Kaztman en particular, la vulnerabilidad se expresa como "...la incapacidad de una persona o de un hogar para aprovechar las oportunidades, disponibles en distintos ámbitos socioeconómicos, para mejorar su situación de bienestar o impedir su deterioro" $(1,2)$. También, se utiliza el concepto de vulnerabilidad para detectar grupos en situación de riesgo por consumo de sustancias, mala alimentación, poca formación educativa o por estar al margen de la ley, o aquellos con mayores posibilidades de sufrir daño o que tienen una condición común por edad, sexo o ubicación, entre otros (3).

La relación directa con la pobreza medida con base en los ingresos monetarios, ha sido, probablemente, el principal enfoque en el desarrollo de los estudios sobre vulnerabilidad. La condición de pobreza determinada por los ingresos, se refiere a la carencia de activos tales como el patrimonio físico, el ahorro financiero, el capital social e, incluso, algunas pautas de comportamiento, entre otros. En este sentido, tanto las comunidades como los hogares y las personas pueden ser vulnerables $(2,3)$.

Es difícil entender y establecer los factores que explican las razones por las que algunas personas, comunidades y grupos tienen mayor capacidad que otros para enfrentar situaciones desfavorables (4). En la vejez aparecen condiciones que generan desventajas o debilidades (5) y, en este grupo de población, la vulnerabilidad social se ha abordado mediante la detección de los grupos de alto riesgo, por ejemplo, los pobres, dependientes 0 aislados (6).

En las personas de edad avanzada, la vulnerabilidad también puede abordarse según el sexo, pues las mujeres presentan mayor riesgo que los hombres

\footnotetext{
Correspondencia:

Doris Cardona, Grupo de Investigación en Epidemiología y Bioestadística, Universidad CES, Calle 10 № 22-04, Medellín, Colombia

Teléfono (574) 444 0555, extensión 1445

dcardona@ces.edu.co

Recibido: 10/04/17; aceptado: 28/08/17
}

ya que cuentan con menos recursos internos y externos (ingresos, estudios, empleo, jubilación, cobertura social, y redes de apoyo social y asistencia) para enfrentar los riesgos y cambios inesperados en la vida (7).

Algunas investigaciones resaltan que aquellos hogares cuyo principal proveedor es el adulto mayor, están más expuestos a las amenazas relacionadas con su pérdida, como la desatención del cuidado de los nietos y de las personas ancianas dependientes, y la dispersión de los miembros del hogar $(5,8)$. Además, con la edad viene la pérdida de capacidades funcionales (9) y mentales (10), lo que incrementa la vulnerabilidad.

En general, la vulnerabilidad aparece ante la carencia de activos en la esfera de los denominados capitales físico, social y humano (2), condiciones que ya se han empleado para construir índices del riesgo de vulnerabilidad por maltrato (11).

Kaztman define los activos como "el conjunto de recursos, materiales e inmateriales, sobre los cuales los individuos y los hogares poseen control y cuya movilización permite mejorar su situación de bienestar, evitar el deterioro de sus condiciones de vida, o bien, disminuir su vulnerabilidad" (2).

El capital humano comprende el estado de salud, las destrezas y las habilidades, y los aspectos mentales relacionados con las motivaciones, las creencias y las actitudes. En los hogares se incluyen el trabajo, y los atributos educativos y de salud de cada miembro. Además, se consideran características relacionadas con los hábitos, como el consumo de sustancias psicoactivas legales e ilegales, así como las actividades realizadas en el tiempo libre, la salud mental relacionada con el riesgo de depresión, la conducta suicida y la afición al juego. La salud física incluye el haberse sentido enfermo, o haber padecido alguna enfermedad en los cuatro meses anteriores, consultar a un profesional por estas dolencias, haber recibido alguna vacuna en el último año, haber recurrido a algún mecanismo legal para ejercer sus derechos, la calidad de la dentadura, la limitación o la discapacidad para desempeñarse en la vida diaria, y los aspectos de satisfacción con su calidad de vida y con los que le generan felicidad. 
Por otra parte, el capital físico se ha descrito como los recursos relacionados con los ahorros monetarios, las rentas y el acceso a créditos, acciones y bonos, entre otros, cuyos beneficios se extienden a otros miembros de la familia, y los bienes materiales como la vivienda, los medios de transporte, los animales, las maquinarias, los terrenos y los bienes durables útiles para la reproducción social que, aunque generan menor liquidez, dan mayor estabilidad. Entre estas características, se cuentan aquellas que algunos autores relacionan con la pobreza y que aumentan la vulnerabilidad, principalmente las variables de la vivienda y el acceso a los servicios públicos, las cuales, conjuntamente con la ocupación, los ingresos, los subsidios y la afiliación al sistema de seguridad social en salud y pensiones, proporcionan seguridad económica a las personas mayores.

El capital social se refiere a las relaciones con otras personas y a la capacidad de movilizarlos hacia el logro de metas individuales y colectivas (2). Dicho capital incluye características como la posición en el hogar, la familia y el grupo de amigos, así como las relaciones con los demás, incluidos los cuidadores, el lugar que se ocupa en el hogar, el acompañamiento para realizar actividades, las personas con las que se convive, el funcionamiento familiar, el número de personas de su red social y familiar, y la percepción de maltrato y discriminación.

A partir de estos criterios, la información empírica ha demostrado suficientemente $(12,13)$ que los adultos mayores dependientes, debido a sus limitaciones funcionales para las actividades básicas cotidianas, tienen mayor riesgo físico y mental frente a la vulnerabilidad. El capital funcional es la capacidad para desarollar actividades de forma autónoma e independiente; por lo tanto, implica un proceso individual, dinámico y cambiante que determina el desempeño funcional del individuo en las actividades más básicas y en las más complejas. En los adultos mayores, las características diferenciadoras son la periodicidad, la frecuencia y la intensidad de la actividad física; las actividades básicas como comer, bañarse, vestirse y asearse; las actividades instrumentales como el uso independiente del teléfono y del transporte público, hacer las compras, preparar y servir la comida, tomar adecuadamente los medicamentos y manejar su propio dinero, así como otras actividades físicas para fortalecer la fuerza muscular y la flexibilidad.
Mediante la medición de estas características, se buscó construir un índice de vulnerabilidad que contribuya a la detección oportuna del riesgo derivado del estado de salud física y mental, así como de los aspectos sociales y humanos.

\section{Materiales y métodos}

Se hizo un estudio transversal cuantitativo con fuentes de información primaria en una población de adultos mayores encuestados por personal capacitado por los investigadores del grupo.

La población de referencia incluía 579.647 personas (expandida a 580.423; error muestral de 2,24\%) de 60 o más años, residentes en las zonas urbanas de Medellín (391.429), Barranquilla (146.445) y Pasto (42.271) en el 2016, según las proyecciones poblacionales del Departamento Administrativo Nacional de Estadística (14). La población de estudio estuvo constituida por una muestra probabilística de 1.514 adultos de ese rango de edad residentes en estas zonas en Medellín (495), Barranquilla (513) y Pasto (506).

El estudio se adelantó en dos etapas. En la primera, se seleccionaron diez barrios de cada comuna o localidad como unidad secundaria de muestreo mediante un muestreo sistemático aleatorio $y$, en la segunda, se seleccionó una manzana de cada barrio como unidad primaria de muestreo mediante la misma técnica. La unidad final de análisis incluyó a todos los adultos mayores residentes en las viviendas ubicadas en cada una de las manzanas seleccionadas.

Se incluyeron adultos de 60 o más años residentes en el área urbana que aceptaran participar y firmar el consentimiento informado, y se excluyeron aquellos con deterioro cognitivo según el Mini-Mental State Examination (MMSE) (15), es decir, con una puntuación inferior a los 13 puntos. También, se excluyeron los adultos mayores que estuvieran bajo el efecto de sustancias psicoactivas.

Las variables se distribuyeron entre las características de los cuatro capitales considerados en la vulnerabilidad (el humano, el físico, el social y el funcional), las cuales se transformaron posteriormente con cuantificación óptima usando el paquete estadístico SPSS ${ }^{\mathrm{TM}}$, versión 21 (licencia otorgada a la Universidad CES).

\section{Análisis estadístico}

Inicialmente, se hizo un análisis univariado con el cálculo de las medidas de frecuencia y las descriptivas, con el fin de caracterizar a los adultos 
mayores según la ciudad de residencia. Se construyó después el índice de vulnerabilidad en el adulto mayor clasificando las variables categóricas después de su cuantificación óptima. Con ellas se corrieron los modelos multivariados mediante un análisis factorial con el método de componentes principales, hasta encontrar el modelo más parsimonioso con mayor varianza explicada. El índice se derivó con una muestra correspondiente al $70 \%$ de los registros de la base original, validada internamente con el $30 \%$ restante de los datos.

La validez de contenido se estableció mediante un análisis factorial exploratorio de los componentes principales con rotación ortogonal varimax, para mejorar la interpretación de los factores extraídos. Se calculó el índice de Kaiser-Meyer-Olkin como medida de adecuación de la muestra. De las 101 variables consideradas al comienzo del análisis (capital humano: 43, físico: 20: social, 21, funcional: 17), se obtuvieron 15 factores, considerando únicamente los ítems con saturaciones factoriales mayores de 0,7 .

Con estos 15 factores, se construyó el modelo general de componentes principales. De nuevo aquí, la validez de contenido se estableció mediante el análisis de la adecuación de la muestra con el índice de Kaiser-Meyer-Olkin, con un resultado de 0,5 , confirmando así que la muestra era adecuada para el análisis factorial. La prueba de esfericidad de Bartlett permitió el rechazo de la hipótesis nula, con lo cual se constató que las variables no estaban correlacionadas en la población $\left(\chi^{2}=\right.$ $134,061 ; p=0,000)$.

Por último, usando la variable construida y categorizada para vulnerable o no vulnerable, se procedió a determinar los factores asociados mediante la prueba de ji al cuadrado $\left(\chi^{2}\right)$, y de medidas epidemiológicas como la razón de prevalencia (RP) cruda y ajustada, y los intervalos de confianza de $95 \%\left(\mathrm{IC}_{95 \%}\right)$; los valores de $\mathrm{p}$ menores de 0,05 se consideraron estadísticamente significativos.

\section{Consideraciones éticas}

El estudio contó con la aprobación del Comité Institucional de Ética de la Universidad CES.

\section{Resultados}

\section{Características de las personas mayores}

Los participantes en el estudio fueron mayoritariamente mujeres (58,2\%), con una relación de 1:4 con respecto a los hombres. La edad promedio fue de 70,23 años (desviación estándar, $\mathrm{DE}=8,2$ años) y la edad más frecuente fueron 69 años, en tanto que $50 \%$ tenía 68 años o menos (rango intercuartílico, $\mathrm{Rl}=11$ años); la edad máxima fue de 104 años.

Se observó predominio de las personas casadas $(38,2 \%)$, seguidas de las viudas $(25,7 \%)$, las solteras $(18,7 \%)$, las separadas o divorciadas $(8,7 \%)$, y aquellas en unión libre (8,7\%). En cuanto al nivel de escolaridad, se halló que $54,9 \%$ de los adultos mayores había cursado algún grado de educación primaria (5 o menos años de educación formal), 26,6 \% había cursado algún grado de educación secundaria y 7,9 \% había accedido a la educación terciaria, en tanto que 10,6 \% no tenía ningún nivel educativo.

\section{Capital humano}

En este, el índice de Kaiser-Meyer-Olkin fue de 0,506, lo cual confirmó que la muestra era adecuada para el análisis factorial. La prueba de esfericidad de Bartlett permitió rechazar la hipótesis nula que afirmaba que las variables no estaban correlacionadas en la población $\left(\chi^{2}=2.260,37\right.$; $\mathrm{p}=0,000$ ).

En el análisis de componentes principales, se encontró una estructura factorial de cinco dimensiones que explicaron el $78,35 \%$ de la varianza total. A estas dimensiones se les asignó un nombre, atendiendo a la mayoría de variables aglutinadas en cada componente: calidad de vida (dos variables relacionadas con la satisfacción con la vida y la salud), salud oral (dos variables relacionadas con las condiciones de la dentadura, aunque el tener dentadura parcial postiza aportó negativamente al capital), acceso a los servicios de salud (dos variables relacionadas con enfermarse y consultar a profesionales de la salud), salud mental (dos variables relacionadas con visitas a psiquiatras y psicólogos) y hábitos (dos variables relacionadas con el consumo de sustancias psicoactivas).

\section{Capital físico}

En este, también se validó el contenido mediante el análisis de la adecuación de la muestra con el índice de Kaiser-Meyer-Olkin, con un resultado de 0,870, lo cual confirmó que la muestra era adecuada para el análisis factorial. La prueba de esfericidad de Bartlett permitió rechazar la hipótesis nula, según la cual las variables no estaban correlacionadas en la población $\left(\chi^{2}=13.613,77 ; p=0,000\right)$.

También, se analizaron los componentes principales y se encontró una estructura factorial de tres dimensiones que explicaron el $77,88 \%$ de la 
varianza total. Los componentes principales se denominaron como: acceso a los servicios públi$\cos$ en la vivienda (siete variables relacionadas con los servicios de energía, acueducto, gas, internet y recolección de basuras); la ocupación (dos variables, aunque la ocupación en el sector informal de la economía aportó de forma negativa al capital físico) y poseer vivienda.

\section{Capital social}

En este, el índice de Kaiser-Meyer-Olkin fue de 0,512 , lo que confirmó que la muestra era adecuada para el análisis factorial. La prueba de esfericidad de Bartlett permitió rechazar la hipótesis nula, según la cual las variables no estaban correlacionadas en la población $\left(\chi^{2}=978,73 ; p=0,000\right)$.

La estructura factorial encontrada fue de cuatro dimensiones que explicaron el $70,64 \%$ de la varianza total. Los componentes encontrados en este capital fueron el acompañamiento para realizar actividades físicas; la violencia intrafamiliar asociada con la disfuncionalidad familiar y una mala percepción de las relaciones que afectó de forma negativa el componente; la convivencia familiar, y la sensación de rechazo en el entorno.

\section{Capital funcional}

En este, la adecuación de la muestra también se analizó mediante el índice de Kaiser-Meyer-Olkin, con un resultado de 0,796 , lo que equivale a que la muestra era adecuada para el análisis factorial. La prueba de esfericidad de Bartlett permitió rechazar la hipótesis nula $\left(\chi^{2}=7.906,93 ; p=0,000\right)$.

Se encontró una estructura factorial de tres dimensiones que explicaron el $75,74 \%$ de la varianza total. Los componentes que emergieron fueron la autonomía del adulto para realizar las actividades instrumentales de la vida diaria (hacer compras, tomar sus medicamentos, usar el transporte público, manejar sus asuntos económicos y preparar sus propias comidas) y el autocuidado relacionado con la capacidad de realizar actividades básicas de la vida diaria, como bañarse, vestirse y desvestirse, usar el sanitario y encargarse de su aseo personal, así como controlar esfínteres. Llama la atención que en este capital solo quedó incluida la independencia para realizar actividades funcionales y no así las variables relacionadas con la actividad física.

En la construcción del modelo final, se incluyeron los siguientes 15 componentes distribuidos en los cuatro capitales, con los cuales se construyó el índice de vulnerabilidad de los adultos mayores en las tres ciudades estudiadas.
1. Capital humano, cinco componentes con diez variables: calidad de vida (dos variables), salud oral (dos variables), acceso a los servicios de salud (dos variables), salud mental (dos variables), y hábitos (dos variables).

2. Capital físico, tres componentes con 11 variables: servicios públicos (siete variables), ocupación (dos variables) y poseer vivienda (dos variables).

3. Capital social, cuatro componentes con ocho variables: acompañamiento (dos variables), violencia intrafamiliar (dos variables), convivencia (dos variables) y discriminación (dos variables).

4. Capital funcional, tres componentes con 11 variables: autonomía (cinco variables), cuidado propio (cuatro variables) y control de esfínteres (dos variables).

Para el modelo global, también se analizó la adecuación de la muestra con el índice de KaiserMeyer-Olkin $(0,5)$ y el rechazo de la hipótesis nula mediante la prueba de esfericidad de Bartlett $\left(\chi^{2}=188,479 ; p=0,000\right)$. Se encontró una estructura factorial con las siguientes tres dimensiones que explicaron el $58,55 \%$ de la varianza total (cuadro 1 ).

1. Uso del tiempo: conformado por un componente del capital humano que incluyó los hábitos de consumo de cigarrillo y alcohol, y otro del capital físico relacionado con la ocupación económica informal o con ser ama de casa.

2. Independencia funcional: conformado por un componente del capital funcional relacionado con la autonomía para desempeñar actividades instrumentales de la vida diaria (uso del transporte público, manejo de los propios asuntos económicos, preparar y servir sus comidas con independencia, tomar los medicamentos y realizar las compras), y otro componente relacionado con la calidad de vida, el cual evidenciaba que cuanto mayor era la satisfacción con la vida y la salud, menor era la vulnerabilidad, es decir, una relación inversamente proporcional.

3. Bienestar subjetivo: conformado por dos componentes, uno de salud mental, que incluyó el riesgo de padecer depresión, ansiedad, conducta suicida, afición al juego y deterioro cognitivo, y el otro relacionado con el hecho de que contar con compañía para salir y realizar algunas actividades disminuía la vulnerabilidad.

\section{Factores asociados con la vulnerabilidad}

A partir de los coeficientes de estos factores, se construyó la escala de vulnerabilidad con dos 
Cuadro 1. Componentes principales de la vulnerabilidad de los adultos mayores en Medellín, Barranquilla y Pasto, 2016

\begin{tabular}{|c|c|c|c|}
\hline \multirow[t]{2}{*}{ Vulnerabilidad del adulto mayor } & \multicolumn{3}{|c|}{ Componentes principales del índice } \\
\hline & Uso del tiempo & Independencia funcional & Bienestar subjetivo \\
\hline $\begin{array}{l}\text { Ocupación (CF) } \\
\text { (Ocupación y empleo) }\end{array}$ & 0,794 & & \\
\hline $\begin{array}{l}\text { Hábitos }(\mathrm{CH}) \\
\text { (Consumo de cigarrillo y alcohol) }\end{array}$ & $-0,793$ & & \\
\hline $\begin{array}{l}\text { Calidad de vida }(\mathrm{CH}) \\
\text { (Satisfacción con la vida y la salud) }\end{array}$ & & $-0,740$ & \\
\hline $\begin{array}{l}\text { Autonomía (CFu) } \\
\text { (Actividades instrumentales de la vida diaria) }\end{array}$ & & 0,739 & \\
\hline $\begin{array}{l}\text { Salud mental }(\mathrm{CH}) \\
\quad \text { (Visita a psiquiatra y psicólogo) }\end{array}$ & & & 0,756 \\
\hline $\begin{array}{l}\text { Acompañamiento (CS) } \\
\text { (Para realizar actividad física) }\end{array}$ & & & $-0,716$ \\
\hline
\end{tabular}

$\mathrm{CH}$ : capital humano; CF: capital físico; CS: capital social; CFu: capital funcional

niveles: aquellas personas que no registraron coeficientes negativos se consideraron como "no vulnerables" y aquellas que presentaron algún coeficiente negativo se denominaron "vulnerables". Así, se encontró que el $20,7 \%$ de las personas mayores no registraban vulnerabilidad, pero el restante $79,3 \%$ sí. Por ciudad, las personas mayores residentes en Pasto fueron las que registraron mayor vulnerabilidad (86,3\%), comparadas con las residentes en las otras dos ciudades estudiadas, estableciéndose diferencias estadísticamente significativas $\left(\chi^{2}=33,375 ; p=0,000\right)$ (cuadro 2).

La mayoría de las personas vulnerables eran de sexo femenino, con edades entre los 60 y los 74 años, casadas, con un nivel educativo de primaria y residentes en viviendas de estrato 2 . La probabilidad de ser vulnerable fue mayor en quienes residían en Pasto, comparados con los de Medellín, en los hombres con respecto a las mujeres, en los longevos (entre 90 y 99 años de edad), en quienes vivían en unión libre, en quienes habían terminado solo la primaria con respecto a los que tenían educación terciaria, en los padres jefes de hogar y en quienes pertenecían al estrato socioeconómico 2 (cuadro 2 ).

Las variables estadísticamente significativas asociadas con la condición de vulnerabilidad, fueron la ciudad, el sexo, el nivel educativo y el rol en el hogar (cuadro 2). Según las escalas calculadas en el estudio, es evidente que todas se asociaron estadísticamente con la vulnerabilidad: tener escaso apoyo social $(R P=2,4)$, vivir en una familia muy disfuncional $(\mathrm{RP}=4,0)$, tener riesgo de depresión $(\mathrm{RP}=18,2)$, tener riesgo de ansiedad $(\mathrm{RP}=1,8)$, presentar algún problema de salud mental $(R P=1,6) 0$ haber sido maltratado $(\mathrm{RP}=2,0)$ (cuadro 3 ).
Por último, al ajustar el modelo explicativo por todas las variables estadísticamente significativas asociadas con la vulnerabilidad, se observó que las siguientes condiciones aumentaban la probabilidad de ser vulnerable: ser residente en Pasto, ser hombre en el rol de padre y jefe del hogar, y tener riesgo establecido de depresión. Las demás categorías perdieron la significación estadística, pero constituyen condiciones que, de todas maneras, aumentaban la probabilidad de presentar vulnerabilidad (cuadro 4).

\section{Discusión}

En este estudio se construyó y se validó internamente un índice de vulnerabilidad de los adultos mayores en tres ciudades de Colombia, a partir de características implícitas en los capitales físico, social, humano y funcional, y se determinaron los factores asociados en un marco conceptual de la vulnerabilidad entendida como el resultado de la interacción de características y de relaciones entre los riesgos sociales (amenazas y exposiciones) y los activos (capacidades de afrontamiento) en contextos específicos $(16,17)$.

Se usó el concepto de vulnerabilidad de Katzman (2), y se agregó un cuarto capital (funcional) a los tres capitales propuestos (humano, físico y social), dada la información comprobada sobre el aporte de la autonomía y la independencia funcional en la calidad de vida.

En diversos estudios se resaltan algunos de los factores que inciden en la vulnerabilidad social de las poblaciones mayores de 60 años, entre ellos, la edad, el sexo y el nivel de estudios (18). Variables como ser hombre y el nivel educativo, se asociaron con la condición de vulnerabilidad. A pesar de que 
al ajustar el nivel educativo por las demás variables este perdió fuerza en la asociación, se pudo establecer que a menor nivel educativo, mayor es la vulnerabilidad. En este sentido, más de la mitad de la población (55\%) del estudio tenía cinco años de educación formal o menos.

En cuanto al capital humano, se consideró la salud mental de la persona mayor, incluidas condiciones como la depresión, la ansiedad y el deterioro cognitivo, pero el riesgo de depresión fue el único que se asoció estadísticamente con la vulnerabilidad, aumentando a medida que se pasaba de un riesgo moderado a uno más alto. A nivel mundial ha cobrado gran relevancia la presencia de la depresión. En un estudio realizado en Arabia Saudí, se reportó una prevalencia de $39 \%$ en personas mayores de 60 años en una muestra comunitaria (19); en España, en una población de mayores de 64 años, la prevalencia se ubicó en 19,7\% (20), y en Alemania, el riesgo de depresión se detectó en 9,7\% de las personas mayores de 75 años sin demencia (21).

En Colombia, según la Encuesta Nacional de Demografía y Salud de 2010, la prevalencia de depresión fue de 9,5\% (22), en tanto que, en el último estudio sobre salud mental en el 2015 (23), se reportó que en las personas mayores de 45 años la prevalencia de cualquier trastorno depresivo se situaba en $5 \%$, siendo inferior a la encontrada en el presente estudio, en el que el riesgo de depresión fue de 7,6\%. En la comparación por ciudades, se encontró que en Pasto la prevalencia del riesgo fue de $28 \%$, es decir, uno de cada tres adultos mayores entrevistados en esta ciudad tenía la probabilidad de sufrir este padecimiento mental, prevalencia similar al $26,3 \%$ encontrado en Bogotá en el 2013 (24).

Cuadro 2. Distribución absoluta y porcentual de los adultos mayores según el índice de vulnerabilidad y las variables sociodemográficas en Medellín, Barranquilla y Pasto, 2016

\begin{tabular}{|c|c|c|c|c|c|c|c|c|c|c|}
\hline \multirow{3}{*}{$\begin{array}{l}\text { Factores } \\
\text { asociados }\end{array}$} & & \multicolumn{4}{|c|}{ Vulnerabilidad } & \multirow[t]{3}{*}{ RPc } & \multirow{2}{*}{\multicolumn{2}{|c|}{$I C_{95 \%}$}} & \multirow[t]{3}{*}{$\chi^{2}$} & \multirow[t]{3}{*}{$\mathbf{p}$} \\
\hline & & \multicolumn{2}{|c|}{ Vulnerable } & \multicolumn{2}{|c|}{ No vulnerable } & & & & & \\
\hline & & $\mathbf{n}$ & $\%$ & $\mathbf{n}$ & $\%$ & & $\mathbf{L i}$ & Ls & & \\
\hline \multirow[t]{3}{*}{ Ciudad } & Medellín & 329 & 28,4 & 133 & 43,9 & 1,00 & - & - & 32,4 & $0,000^{*}$ \\
\hline & Barranquilla & 402 & 34,7 & 102 & 33,7 & 1,59 & 1,19 & 2,14 & & \\
\hline & Pasto & 429 & 37,0 & 68 & 22,4 & 2,55 & 1,84 & 3,53 & & \\
\hline \multirow[t]{2}{*}{ Sexo } & Hombre & 455 & 39,2 & 48 & 15,8 & 3,43 & 2,47 & 4,77 & 53,5 & $0,000^{*}$ \\
\hline & Mujer & 705 & 60,8 & 255 & 84,2 & 1,00 & - & - & 30,0 & 0,000 \\
\hline \multirow[t]{4}{*}{ Grupo de edad } & Joven (60 a 74 años) & 841 & 72,5 & 227 & 74,9 & 1,00 & - & - & 3,4 & 0,330 \\
\hline & Viejo (75 a 89 años) & 294 & 25,3 & 75 & 24,8 & 1,06 & 0,79 & 1,42 & & \\
\hline & $\begin{array}{l}\text { Longevo (90 a } 99 \\
\text { años) }\end{array}$ & 24 & 2,1 & 1 & 0,3 & 6,48 & 0,87 & 48,14 & & \\
\hline & Centenario & 1 & 0,1 & 0 & 0,0 & $\mathrm{NC}$ & $\mathrm{NC}$ & NC & & \\
\hline \multirow[t]{5}{*}{ Estado civil } & Soltero & 185 & 15,9 & 50 & 16,5 & 1,00 & - & - & 2,1 & 0,724 \\
\hline & Casado & 468 & 40,3 & 126 & 41,6 & 1,00 & 0,69 & 1,45 & & $0, r<4$ \\
\hline & Unión libre & 120 & 10,3 & 23 & 7,6 & 1,41 & 0,82 & 2,43 & & \\
\hline & Separado/divorciado & 102 & 8,8 & 28 & 9,2 & 0,99 & 0,58 & 1,66 & & \\
\hline & Viudo & 285 & 24,6 & 76 & 25,1 & 1,01 & 0,68 & 1,52 & & \\
\hline \multirow[t]{4}{*}{ Nivel educativo } & Ninguno & 114 & 9,8 & 26 & 8,6 & 1,67 & 0,95 & 2,96 & 10,8 & $0,013^{*}$ \\
\hline & Primaria & 637 & 54,9 & 140 & 46,2 & 1,74 & 1,14 & 2,64 & & \\
\hline & Secundaria & 312 & 26,9 & 100 & 33,0 & 1,19 & 0,77 & 1,85 & & \\
\hline & Terciaria & 97 & 8,4 & 37 & 12,2 & 1,00 & - & - & & \\
\hline \multirow[t]{5}{*}{ Rol en el hogar } & Jefe de hogar & 745 & 64,3 & 172 & 56,8 & 1,48 & 0,82 & 2,66 & 25,35 & $0,000^{*}$ \\
\hline & Cónyuge del jefe & 185 & 16,0 & 81 & 26,7 & 0,78 & 0,42 & 1,45 & & \\
\hline & Padre del jefe & 142 & 12,3 & 20 & 6,6 & 2,42 & 1,16 & 5,04 & & \\
\hline & Hermano del jefe & 40 & 3,5 & 14 & 4,6 & 0,97 & 0,42 & 2,24 & & \\
\hline & Otro & 47 & 4,1 & 16 & 5,3 & 1,00 & - & - & & \\
\hline \multirow{6}{*}{$\begin{array}{l}\text { Estrato socio- } \\
\text { económico }\end{array}$} & 1 & 312 & 26,9 & 76 & 25,1 & 1,03 & 0,11 & 9,32 & 7,6 & 0,179 \\
\hline & 2 & 472 & 40,7 & 112 & 37,0 & 1,05 & 0,12 & 9,52 & 1,0 & 0,170 \\
\hline & 3 & 301 & 26,0 & 84 & 27,7 & 0,90 & 0,10 & 8,12 & & \\
\hline & 4 & 49 & 4,2 & 18 & 5,9 & 0,68 & 0,07 & 6,50 & & \\
\hline & 5 & 21 & 1,8 & 12 & 4,0 & 0,44 & 0,04 & 4,38 & & \\
\hline & 6 & 4 & 0,3 & 1 & 0,3 & 1,00 & - & - & & \\
\hline \multirow{2}{*}{$\begin{array}{l}\text { Ingreso económico } \\
\text { en el mes anterior }\end{array}$} & Sí & 650 & 56,1 & 176 & 58,1 & 1,00 & - & - & 0,4 & 0,541 \\
\hline & No & 508 & 43,9 & 127 & 41,9 & 1,08 & 0,84 & 1,40 & & \\
\hline
\end{tabular}

*RPc: razón de prevalencia cruda; NC: no se calculó; IC: intervalo de confianza; Li: límite inferior; Ls: límite superior; *p: valor estadísticamente significativo 
Cuadro 3. Distribución absoluta y porcentual de los adultos mayores según el índice de vulnerabilidad y apoyo social, APGAR (Adaptability, Partnership, Growth, Affection, and Resolve) familiar, y las variables de depresión, ansiedad, salud mental y maltrato en Medellín, Barranquilla y Pasto, 2016

\begin{tabular}{|c|c|c|c|c|c|c|c|c|c|}
\hline \multirow[t]{3}{*}{ Factores asociados } & \multicolumn{4}{|c|}{ Vulnerabilidad } & \multirow[t]{3}{*}{ RPc } & \multirow{2}{*}{\multicolumn{2}{|c|}{$\mathrm{IC}_{95 \%}$}} & \multirow[t]{3}{*}{$\chi^{2}$} & \multirow[t]{3}{*}{$\mathbf{P}$} \\
\hline & \multicolumn{2}{|c|}{ Vulnerable } & \multicolumn{2}{|c|}{ No vulnerable } & & & & & \\
\hline & $\mathbf{n}$ & $\%$ & $\mathbf{n}$ & $\%$ & & $\mathbf{L i}$ & Ls & & \\
\hline \multicolumn{10}{|l|}{ Apoyo social } \\
\hline Adecuado & 1.048 & 90,9 & 287 & 96,0 & 1,0 & - & - & 7,8 & $0,005^{*}$ \\
\hline Escaso & 105 & 9,1 & 12 & 4,0 & 2,4 & 1,3 & 4,4 & & \\
\hline \multicolumn{10}{|c|}{ Disfuncionalidad familiar } \\
\hline Grave & 84 & 7,3 & 7 & 2,3 & 4,0 & 1,8 & 8,7 & 25,7 & $0,000^{*}$ \\
\hline Moderada & 103 & 8,9 & 16 & 5,3 & 2,1 & 1,2 & 3,7 & & \\
\hline Leve & 298 & 25,8 & 57 & 18,9 & 1,7 & 1,3 & 2,4 & & \\
\hline Función normal & 671 & 58,0 & 222 & 73,5 & 1,0 & - & - & & \\
\hline \multicolumn{10}{|l|}{ Riesgo de depresión } \\
\hline Sin depresión & 979 & 84,4 & 287 & 95,0 & 1,0 & - & - & 17,9 & $0,000^{*}$ \\
\hline Probable & 119 & 10,3 & 14 & 4,6 & 2,5 & 1,4 & 4,4 & & \\
\hline Establecido & 62 & 5,3 & 1 & 0,3 & 18,2 & 2,5 & 131,7 & & \\
\hline \multicolumn{10}{|l|}{ Riesgo de ansiedad } \\
\hline No & 1.003 & 87,7 & 276 & 92,9 & 1,0 & - & - & 6,4 & $0,012^{*}$ \\
\hline Sí & 141 & 12,3 & 21 & 7,1 & 1,8 & 1,1 & 3,0 & & \\
\hline \multicolumn{10}{|l|}{ Salud mental } \\
\hline Sin problemas & 804 & 70,7 & 233 & 79,8 & 1,0 & - & - & 9,5 & $0,002^{*}$ \\
\hline Deteriorada & 333 & 29,3 & 59 & 20,2 & 1,6 & 1,2 & 2,2 & & \\
\hline \multicolumn{10}{|l|}{ Maltrato } \\
\hline No & 1.030 & 89,3 & 283 & 94,3 & 1,0 & - & - & 6,8 & $0,009^{*}$ \\
\hline Sí & 124 & 10,7 & 17 & 5,7 & 2,0 & 1,2 & 3,4 & & \\
\hline
\end{tabular}

RPc: razón de prevalencia cruda, NC: no calculado; IC: intervalo de confianza; Li: límite inferior; Ls: límite superior

*p: valor estadísticamente significativo

Los trastornos de ansiedad en la población adulta mayor han recibido menor atención que otros y son pocos los estudios sobre la presencia de ansiedad en esta población, lo cual dificulta las comparaciones. Algunos de los datos reportados, de hace una década, muestran prevalencias de ansiedad que oscilan entre 4 y $50 \%$, lo que indica que las estrategias para su atención han sido insuficientes, pues la prevalencia del riesgo de ansiedad en el presente estudio fue cercana al $10 \%$. En un estudio en México, se halló una prevalencia de ansiedad cercana al 50\% en la población mayor de 55 años (25); en Puerto Rico, se encontró una de $6,9 \%$ (26), mientras que en Nueva Zelanda, fue de 3,8\% (27), y en Chile, se encontraron prevalencias entre 7,9 y $13,3 \%$ (28).

En el presente estudio, se presentó riesgo de ansiedad en 9,2\% de los adultos mayores, siendo los residentes en Pasto quienes presentaron la mayor prevalencia $(20,8 \%)$ y los de Barranquilla, la menor $(4,3 \%)$. En cuanto a las diferencias por sexo, se encontró que la prevalencia en las mujeres era mayor que en los hombres, similar a lo reportado en Estados Unidos en un estudio del 2006 en el que se halló que los trastornos de ansiedad eran dos veces más prevalentes en las mujeres (29).
La prevalencia de deterioro cognitivo en este estudio se clasificó como levemente grave, pues entre los criterios de selección debieron excluirse los adultos mayores cuyo deterioro de la capacidad cognitiva les impedía responder a los cuestionarios, $y$, por lo tanto, la prevalencia fue de $4 \%$, similar a la reportada por Mejía, et al., en México (30), pero menor a la registrada en el Estudio Nacional de Salud, Bienestar y Envejecimiento (SABE) (23) realizado en Colombia en el 2015, en el cual se reportó una prevalencia de deterioro cognitivo leve de $17 \%$.

Se observó que la frecuencia de deterioro cognitivo era más alta en el sexo masculino, aunque la asociación no fue significativa, al igual que en el estudio de Escobar, et al., en Perú (31). El predominio del deterioro cognitivo en hombres se ha documentado ampliamente en estudios nacionales e internacionales (31-34); sin embargo, algunas de las diferencias reportadas con relación al sexo podrían deberse a las distribuciones socioculturales de las poblaciones. Se encontró una clara asociación entre el deterioro cognitivo y la edad, con mayor predominio $(60,8 \%)$ en los adultos mayores entre 75 y 89 años de edad en las tres ciudades, lo cual coincide con lo encontrado en Perú (31), México (32), Cuba (35) y Chile 
Cuadro 4. Razón de prevalencia de la vulnerabilidad en el adulto mayor según los factores asociados, 2016

\begin{tabular}{|c|c|c|c|c|c|c|c|c|c|}
\hline \multirow[t]{2}{*}{ Variable } & & \multirow[t]{2}{*}{ RPc } & \multicolumn{2}{|c|}{$\mathrm{IC}_{95 \%}$} & \multirow[t]{2}{*}{$\mathrm{RPa}$} & \multicolumn{2}{|c|}{$\mathrm{IC}_{95 \%}$} & \multirow[t]{2}{*}{$\chi^{2}$} & \multirow[t]{2}{*}{$\mathbf{p}$} \\
\hline & & & Li & Ls & & Li & Ls & & \\
\hline \multirow[t]{3}{*}{ Ciudad } & Medellín & 1,000 & - & - & 1,000 & - & - & 27,2 & $0,000^{*}$ \\
\hline & Barranquilla & 1,593 & 1,2 & 2,143 & 2,0 & 1,45 & 2,86 & & \\
\hline & Pasto & 2,55 & 1,8 & 3,532 & 2,3 & 1,62 & 3,36 & & \\
\hline \multirow[t]{2}{*}{ Sexo } & Hombre & 3,429 & 2,5 & 4,77 & 3,6 & 2,48 & 5,17 & 46,5 & $0,000^{*}$ \\
\hline & Mujer & 1,000 & - & - & 1,000 & - & - & & \\
\hline \multirow[t]{4}{*}{ Nivel educativo } & Ninguno & 1,672 & 0,9 & 2,957 & 1,7 & 0,85 & 3,22 & 7,2 & 0,065 \\
\hline & Primaria & 1,736 & 1,1 & 2,643 & 1,8 & 1,09 & 2,89 & & \\
\hline & Secundaria & 1,19 & 0,8 & 1,849 & 1,2 & 0,76 & 2,04 & & \\
\hline & Terciaria & 1,000 & - & - & 1,000 & - & - & & \\
\hline \multirow[t]{5}{*}{ Rol en el hogar } & Jefe de hogar & 1,48 & 0,82 & 2,66 & 1,5 & 0,77 & 2,87 & 13,0 & $0,011^{*}$ \\
\hline & Cónyuge del jefe & 0,78 & 0,42 & 1,45 & 1,1 & 0,53 & 2,15 & & \\
\hline & Padre del jefe & 2,42 & 1,16 & 5,04 & 2,7 & 1,21 & 6,03 & & \\
\hline & Hermano del jefe & 0,97 & 0,42 & 2,24 & 1,0 & 0,39 & 2,40 & & \\
\hline & Otro & 1,000 & - & - & 1,000 & - & - & & \\
\hline \multirow{6}{*}{$\begin{array}{l}\text { Estrato socio- } \\
\text { económico }\end{array}$} & 1 & 1,03 & 0,11 & 9,32 & 0,6 & 0,06 & 5,48 & 2,8 & 0,733 \\
\hline & 2 & 1,05 & 0,12 & 9,52 & 0,7 & 0,07 & 6,33 & & \\
\hline & 3 & 0,90 & 0,10 & 8,12 & 0,6 & 0,06 & 5,71 & & \\
\hline & 4 & 0,68 & 0,07 & 6,50 & 0,4 & 0,04 & 4,36 & & \\
\hline & 5 & 0,44 & 0,04 & 4,38 & 0,5 & 0,04 & 4,85 & & \\
\hline & 6 & 1,000 & - & - & 1,000 & - & - & & \\
\hline \multirow[t]{2}{*}{ Apoyo social } & Adecuado & 1,000 & - & - & 1,000 & - & - & 0,189 & 0,664 \\
\hline & Escaso & 2,4 & 1,3 & 4,4 & 0,8 & 0,4 & 1,8 & & \\
\hline \multirow{4}{*}{$\begin{array}{l}\text { Disfuncionalidad } \\
\text { familiar }\end{array}$} & Grave & 4,0 & 1,8 & 8,7 & 2,2 & 0,8 & 5,8 & 5,928 & 0,115 \\
\hline & Moderado & 2,1 & 1,2 & 3,7 & 1,5 & 0,8 & 2,8 & & \\
\hline & Leve & 1,7 & 1,3 & 2,4 & 1,4 & 1,0 & 2,1 & & \\
\hline & Familia funcional & 1,000 & - & - & 1,000 & - & - & & \\
\hline \multirow[t]{3}{*}{ Riesgo de depresión } & Sin depresión & 1,000 & - & - & 1,000 & - & - & 8,687 & $0,013^{*}$ \\
\hline & Probable & 2,5 & 1,4 & 4,4 & 2,0 & 1,0 & 4,2 & & \\
\hline & Establecida & 18,2 & 2,5 & 131,7 & 13,8 & 1,8 & 107,7 & & \\
\hline \multirow[t]{3}{*}{ Riesgo de ansiedad } & No & 1,000 & - & - & 1,000 & - & - & 0,932 & 0,334 \\
\hline & Sí & 1,8 & 1,1 & 3,0 & 0,7 & 0,4 & 1,4 & & \\
\hline & Sin problemas & 1,0 & & & 1,0 & & & 0,045 & 0,832 \\
\hline Salud mental & Deteriorada & 1,6 & 1,2 & 2,2 & 1,1 & 0,7 & 1,7 & & \\
\hline \multirow[t]{2}{*}{ Maltrato } & No & 1,000 & - & - & 1,000 & - & - & 0,608 & 0,435 \\
\hline & Sí & 2,0 & 1,2 & 3,4 & 1,3 & 0,7 & 2,3 & & \\
\hline
\end{tabular}

${ }^{*} \mathrm{RPc}$ : razón de prevalencia cruda; RPa: razón de prevalencia ajustada; NC: no se calculó; IC: intervalo de confianza; Li: límite inferior; Ls: límite superior; * $p$ : valor estadísticamente significativo

(36). En la mayoría de los estudios consultados, se señala una importante relación entre la edad y el funcionamiento cognitivo, explicable por los cambios del metabolismo a medida que avanza la edad, lo que se traduce en una disminución de la entrada de glucosa y la recaptación de glutamato extracelular con la consecuente muerte de neuronas en el hipocampo. Si a ello se añaden los componentes del estilo de vida, las probabilidades de un mayor deterioro cognitivo a medida que se envejece es más alta (37).

Una posible limitación en el estudio de la capacidad cognitiva fue el instrumento de medición, ya que se usó uno diseñado para la detección, mas no para el diagnóstico del deterioro cognitivo grave, aunque sí permite detectar a quienes requerirían una posterior evaluación con una batería neuropsicológica de diagnóstico aplicada por personal especializado.

En cuanto al capital social, el apoyo con el que las personas cuentan constituye una alternativa con efectos positivos en diferentes ámbitos de la vida, sobre todo en la dinámica familiar (38). En el presente estudio, se evidenció que $6 \%$ de los adultos mayores tenía poco apoyo de su entorno social, y 90,6\% de este porcentaje pertenecía a familias disfuncionales. Con el correr de los años, las limitaciones y el nivel de dependencia de los adultos mayores implican que tengan que vivir con sus hijos $u$ otros familiares, en hogares en los que generalmente predomina la tipología familiar 
extensa (39-41). En este sentido, se encontró que en Medellín y Barranquilla más de la mitad de los adultos mayores pertenecía a familias extensas, en tanto que en Pasto predominaron las familias nucleares. Al igual que en otros estudios, en este también se encontró que los adultos mayores percibían su familia como funcional (39).

En cuanto al problema del maltrato del adulto mayor en las familias, este ya se considera como un problema de salud pública muy subestimado a nivel mundial (42-44). En este estudio, se registró una prevalencia de maltrato de $7,1 \%$ en las tres ciudades, siendo mayor en Pasto (13\%) que en las otras dos ciudades. Dicha prevalencia es superior a la encontrada en países desarrollados (alrededor del $4 \%$ ) (45). Según datos del Instituto Nacional de Medicina Legal y Ciencias Forenses, entre el 2004 y el 2011, alrededor de 10.000 adultos mayores fueron víctimas de alguna agresión en el país (46). En una revisión sistemática, se encontró que, según los estudios en población general, $6 \%$ de las personas reportó maltrato (47). Entre las prevalencias más altas se encuentra la registrada en un área rural de Egipto (43,7\%), siendo la negligencia la forma más frecuente (42), en tanto que, entre las prevalencias más bajas, está la reportada en España en el 2008 (0,8 \%) (48). En México, en la Encuesta sobre Maltrato a Personas Mayores (EMPAM-DF) del 2006, se registró una prevalencia de maltrato de 16,2\% (49) y, en un estudio del 2012 en adultos mayores que no residían en instituciones, se encontró una prevalencia de maltrato de $10,3 \%$.

Como se ha reportado en diversos estudios $(48,50$ 52 ), en el nuestro el porcentaje de maltrato en las mujeres fue mayor que en los hombres $(48,53)$. En cuanto a los recursos económicos, se observó que el porcentaje de personas maltratadas fue mayor entre quienes contaban con algún ingreso, lo cual se contrapone a los resultados de otros estudios, en los que los adultos mayores sin ingresos económicos eran más propensos al maltrato (42). Se encontró que $15,6 \%$ de los adultos mayores maltratados correspondía a aquellos que dependían de otras personas para realizar actividades básicas de la vida diaria, aunque la asociación no fue significativa. En otros estudios se ha determinado la dependencia como un factor de riesgo para el maltrato.

Con respecto a la capacidad funcional de los adultos mayores, en el presente estudio se encontró que nueve de cada diez personas tenían independencia funcional; los factores asociados a la capaci-dad funcional fueron la edad (adultos entre 60 y 74 años), la actividad física y no tener discapacidad motriz ni riesgo nutricional.

Aunque la evidencia empírica se ha centrado principalmente en los factores asociados con el deterioro de la capacidad funcional $(54,55)$, deben destacarse las características asociadas con la independencia funcional de los adultos mayores en respuesta a los nuevos retos que plantea el envejecimiento activo, lo que exige garantizarles una vida saludable y digna, así como formas de trabajo y otros componentes positivos, como la actividad física, una adecuada nutrición y la prevención de la discapacidad motriz.

Estas características concuerdan con las reportadas en estudios recientes, en los cuales se ha constatado que factores demográficos, como la edad, los hábitos de vida y el cuidado de la salud, están asociados con la independencia funcional $(11,55$ 57). Aunque en este estudio se registró una prevalencia alta de adultos mayores con independencia funcional, hubo una diferencia significativa entre los adultos mayores más jóvenes (de 60 a 74 años) y aquellos con 75 o más años, lo cual concuerda con los resultados de la reciente encuesta nacional SABE, en la cual la dependencia fue menor de $20 \%$ en el grupo etario de 60 a 74 años, comparado con el grupo de 85 o más años (más de 40\%) (23). En este sentido, en estudios recientes en Brasil, se halló una asociación entre el deterioro de la capacidad funcional y la edad, el sedentarismo, la presencia de enfermedades y la inactividad física (12).

En cuanto al riesgo nutricional, se ha documentado que el bienestar nutricional es un factor determinante de la capacidad funcional, la independencia y la calidad de vida de los adultos mayores $(54,55,58,59)$. En un estudio reciente en Japón, se encontró que, a mayor riesgo nutricional, mayor deterioro de la capacidad funcional; la proporción de independencia funcional de los adultos sin riesgo nutricional fue de $98,1 \%$, en tanto que, en aquellos con riesgo nutricional alto, esta proporción se redujo a 87,8 \% (54). En otro estudio en esta misma región, se había encontrado previamente que la dependencia funcional de los adultos mayores de tres grupos étnicos rurales era mayor del $20 \%$ y que factores como el índice de masa corporal, la reducción del apetito y el número de comidas diarias, constituían factores de predicción del estado de la capacidad funcional de este grupo poblacional (59). Aunque en el presente 
estudio no se indagó por el tipo de alimentación, al ajustar por las variables de predicción, se encontró que, por cada dos personas sin riesgo nutricional e independencia funcional, solo una con riesgo nutricional presentaba también independencia.

Se sabe que la actividad física es beneficiosa para la salud, pues mejora las funciones respiratorias, el estado muscular y el óseo, y repercute positivamente en la capacidad funcional de los adultos mayores, previniendo, además, el deterioro cognitivo y de la salud mental. La mortalidad por enfermedades crónicas en las personas mayores que realizan algún tipo de actividad física es menor y su estado cardiovascular es mejor, en comparación con los menos activos (60). Aunque en algunos estudios se ha encontrado una asociación entre la capacidad funcional y otros factores demográficos, sociales, de salud, de hábitos y de mala percepción de la propia salud (61), que no se registró en este estudio, sí se encontraron diferencias proporcionales en la capacidad funcional según la categoría de dichas variables. La ausencia de asociación puede explicarse por la poca heterogeneidad en las variables sociales y de percepción que no fueron incluidas en el modelo final. También, se encontró que variables como la calidad de vida y la participación comunitaria, que registraron una significación importante en el análisis bivariado, no aparecieron como factores asociados en el modelo final, quizá debido a la homogeneidad en las respuestas.

Como lo plantean algunos autores, la vulnerabilidad está relacionada con la pobreza y, por eso, es necesario establecer las condiciones económicas de las personas mayores. En este sentido, se encontró que cuatro de cada diez personas mayores requerían del apoyo económico de familiares, amigos u organizaciones. Dicha dependencia económica puede llevarlos a la pobreza, principalmente a las mujeres, pues más de la mitad $(54,3 \%)$ de ellas no había percibido ingresos en el mes anterior a la encuesta, con las implicaciones que esto tiene para personas cuya edad les limita el acceso al empleo formal. Esta falta de ingresos indica que otro componente del índice de vulnerabilidad relacionado con el uso del tiempo y la ocupación, es la necesidad de tener un empleo más formal que garantice a las personas mayores un ingreso económico más constante.

El otro componente de la vulnerabilidad fue la independencia funcional frente a las actividades de la vida diaria. Las características relacionadas con dicha independencia constituyen una red compleja, por lo cual deben reorientarse las acciones para promover mejor salud y calidad de vida en los adultos mayores, potenciando su capacidad funcional.

Una posible limitación del estudio serían los sesgos de información en la medición de algunas de las variables, pues el reporte lo hicieron los propios participantes, quienes no necesariamente tenían información precisa sobre algunas condiciones clínicas, o presentaban vacíos de memoria. Ello explicaría que algunas variables, como la presencia de enfermedad, la percepción de la salud y la calidad de vida, no hayan quedado incluidas en el modelo.

Al construir el índice de vulnerabilidad, se encontró que el bienestar subjetivo aportaba o disminuía la condición de vulnerable, siendo esta mayor en aquellos con riesgo de padecer depresión, ansiedad o deterioro cognitivo

Los resultados obtenidos sirven como base conceptual en el análisis del impacto del envejecimiento poblacional en las comunidades, y aportan elementos para el mejoramiento de la calidad de vida del adulto mayor, principalmente, en aspectos como la capacidad funcional para mantener la independencia el mayor tiempo posible, el fortalecimiento de la salud mental para evitar los riesgos de padecer depresión, ansiedad, deterioro cognitivo, y adquirir hábitos deletéreos que pueden influir en su bienestar subjetivo.

Asimismo, permiten visualizar los requisitos institucionales futuros de los programas de promoción y prevención, y la necesidad de mejorar la cobertura en seguridad social y las condiciones de vida de un grupo poblacional que aumenta día a día sin que las sociedades se percaten ni se preparen adecuadamente para ello.

Este índice constituye un instrumento de detección y seguimiento ideal para los profesionales que atienden esta población, y puede orientar a los responsables de las decisiones en la adopción de estrategias de reducción del riesgo de vulnerabilidad.

\section{Conflicto de intereses}

Los autores declaran no tener conflictos de intereses.

\section{Financiación}

Colciencias (código 122871149710) y Universidad CES.

\section{Referencias}

1. González L. Vulnerabilidad socio-demográfica en el Gran Córdoba. Diagnóstico y perspectivas en el período 2001-16. Fecha de consulta:28 de marzo de 2016. Disponible en: http:// www.redaepa.org.ar/jornadas/ixjornadas/resumenes/Se27-Prospectiva_Arriaga/GonzalezVulnerabilidad2001_16.pdf 
2. Kaztman R. Notas sobre la medición de la vulnerabilidad social. Santiago de Chile: CEPAL; 2000. p. 275-301.

3. Organización de las Naciones Unidas, Comisión Económica para América Latina y el Caribe. Vulnerabilidad sociodemográfica: viejos y nuevos riesgos para comunidades, hogares y personas. Fecha de consulta: 26 de julio de 2017. Disponible en: http://www.cepal.org/ publicaciones/xml/4/11674/LCW3-Vulnerabilidad.pdf

4. Sánchez D. Contexto ambiental y experiencia espacial de envejecer en el lugar: el caso de Granada. Pap Poblac. 2009;15:175-213.

5. Sánchez WB. Vulnerabilidad socio-demográfica de las personas de la tercera edad. Estudio de caso, centro histórico de La Habana. Cuadernos Geográficos. 2013;52:153-77.

6. Bazo MT. Consecuencias del envejecimiento en la sociedad española actual. En: Kristensen B, Álvarez Pousa L, Evans Pim J, editores. Comunicación e pessoas maiores. Actas do Foro Internacional. Santiago de Compostela: Colexio Profesional de Xornalistas de Galicia; 2007. p. 239-56.

7. González C, Ham-Chande R. Funcionalidad y salud: una tipología del envejecimiento en México. Salud Pública Méx. 2007;49:s448-58.

8. González DS. Geografía del envejecimiento vulnerable y su contexto ambiental en la ciudad de Granada: discapacidad, dependencia y exclusión social. Cuadernos Geográficos. 2009;45:107-35.

9. Chatterji S. Health, functioning, and disability in older adults present status and future implications. Lancet. 2014;385: 563-75. https://doi.org/10.1016/S0140-6736(14)61462-8

10. Steptoe A, Deaton A, Stone A. Subjective wellbeing, health, and ageing. Lancet. 2014;385:640-8. https://doi. org/10.1016/S0140-6736(13)61489-0

11. Dong X, Simon MA. Vulnerability risk index profile for elder abuse in a community dwelling. J Am Geriatr Soc. 2014;62:10-5.

12. Ahlqvist A, Nyfors H, Suhonen R. Factors associated with older people's independent living from the viewpoint of health and functional capacity: A register-based study. Nurs Open. 2016;3:79-89. https://doi.org/10.1002/nop2.39

13. Kagawa CA, Corrente JE. Análise da capacidade funcional em idosos do município de Avaré-SP: fatores associados. Rev Bras Geriatr Gerontol. 2015;18:577-86. https://doi. org/10.1590/1809-9823.2015.14140

14. Departamento Administrativo Nacional de Estadística. Proyecciones de población: proyecciones municipales 20052011. Fecha de consulta: 7 de abril de 2016. Disponible en: http://www.dane.gov.co/files/investigaciones/poblacion/ proyepobla06_20/p_20052011_Ajustadosedadessimples 024.xls

15. Icaza M, Albala C. Minimental State Examinations del estudio de demencia en Chile: análisis estadístico. Washington, D.C.: OPS; 1999.

16. Delor F, Hubert M. Revisiting the concept of 'vulnerability'. Soc Sci Med. 2000;50:1557-70. https://doi.org/10.1016/ S0277-9536(99)00465-7

17. Sánchez D. Envejecimiento demográfico urbano y sus repercusiones socioespaciales en México. Retos de la planeación gerontológica. Rev Geogr Norte Gd. 2007;38:4561. https://doi.org/10.4067/S0718-34022007000200003
18. Vázquez DC, González DS. Los ancianos al desván. El proceso de degradación biológica y social de la población mayor del municipio de Granada. Cuadernos Geográficos. 2005;36:255-74.

19. Al-Shammari SA, Al-Subaie A. Prevalence and correlates of depression among Saudi elderly. Int J Geriatr Psychiatry. 1999;14:739-47.

20. Urbina JR, Flores JM, García MP, Torres L, Torrubias RM. Depressive symptoms in the elderly. Prevalence and associated factors. Gac Sanit. 2007;21:37-42.

21. Weyerer S, Eifflaender-Gorfer S, Köhler L, Jessen F, Maier W, Fuchs A. Prevalence and risk factors for depression in non-demented primary care attenders aged 75 years and older. J Affect Disord. 2008;111:153-63. https://doi. org/10.1016/j.jad.2008.02.008

22. Aldana RA, Pedraza JA. Análisis de la depresión en el adulto mayor en la encuesta nacional de demografía y salud 2010. Tesis. Bogotá: Universidad del Rosario; 2012.

23. Ministerio de Salud, Colciencias. SABE Colombia 2015: Estudio Nacional de Salud, Bienestar y Envejecimiento. Fecha de consulta: 27 de julio de 2017. Disponible en: https:// www.minsalud.gov.co/sites/rid/Lists/BibliotecaDigital/RIDE/ VS/ED/GCFI/Resumen-Ejecutivo-Encuesta-SABE.pdf

24. Gómez C, Rodríguez M, Díaz N, Cano C, Tamayo $\mathbf{N}$. Depresión y satisfacción con la vida en personas mayores de 60 años en Bogotá: Encuesta de Salud, Bienestar y Envejecimiento (SABE). Rev Colomb Psiquiat. 2013;43(Supl.1):65-70.

25. Quiroz C, Flores R. Ansiedad y depresión en adultos mayores. Psicología y Salud. 2007;17:291-300.

26. CaninoGJ,BirdHR,ShroutPE,RubioM,Bravo M, Martínez $\mathbf{R}$, et al. The prevalence of specific psychiatric disorders in Puerto Rico. Arch Gen Psychiatry. 1987;44:727-35. https:// doi.org/10.1001/archpsyc.1987.01800200053008

27. Regier DA, Rae DS, Narrow WE, Kaelbert CT, Schatzberg AF. Prevalence of anxiety disorders and their comorbidity with mood and addictive disorders. Br J Psychiatry Suppl. 1998;173:24-8.

28. Antequera-Jurado F. Trastornos de ansiedad en el anciano. Psicogeriatría. 2009;1:37-47.

29. Somers JM, Goldner EM, Waraich P, Hsu L. Prevalence and incidence studies of anxiety disorders. A systematic review of literature. Can J Psychiatry. 2006;51:100-13. https://doi.org/10.1177/070674370605100206

30. Mejía S, Miguel A, Villa A, Ruiz L, Gutiérrez L. Deterioro cognoscitivo y factores asociados en adultos mayores en México. Salud Pública Méx. 2007;49(Supl.4):475-81.

31. Escobar G, Ramos L, Rodríguez C, Montero A, Peñalva J, Valencia K. Deterioro cognitivo leve en adultos mayores pertenecientes a clubes y albergues de la tercera edad de la ciudad de Tacna. Rev Psicol Arequipa. 2013;3:27-38.

32. Ibarra L, Villareal J, Salinas R. Capacidad cognitiva de los pacientes de un servicio geriátrico ambulatorio en Monterrey, México. Med Univer. 2006;8:84-7.

33. Moquillaza M, León E, Dongo M, Munayco C. Características sociodemográficas y de salud de los adultos mayores en situación de calle en Lima, Perú. Rev Peru Med Exp Salud Pública. 2015;32:693-9. 
34. Cerquera AM. Relación entre los procesos cognitivos y el nivel de depresión en las adultas mayores institucionalizadas en el Asilo San Antonio de Bucaramanga. Univ Psychol. 2008;7:271-81

35. Rodríguez JR, Tabares VZ, Jiménez ES, López RS, Del Carmen M. El manejo del adulto mayor. Panor Cuba Salud. 2014;9:35-41.

36. Poblete F, Matus C, Díaz E, Vidal P, Ayala M. Depresión, cognición y calidad de vida en adultos mayores activos. Revista Ciencias de la Actividad Física UCM. 2015;16:71-7.

37. Carlson N. Fisiología de la conducta. Primera edición. Madrid: Editorial Pearson; 2009.

38. Sierra O, Madariaga C, Abello R. Relación entre la pertenencia a redes sociales y dinámica familiar de mujeres trabajadoras en condiciones de pobreza. Psicología desde el Caribe. 1998;1:94-113.

39. Martín P, María M, Delgado A, Ferregut N, Augusto J, Álvarez K, et al. Medio familiar de los adultos mayores. Rev Cienc Médicas Pinar Río. 2012;16:97-104.

40. Herrera PM. Jubilación y vejez, su repercusión en la salud familiar. Rev Cubana Med Gen Integr. 2008;24:1-7.

41. Ramos EF. Caracterización del riesgo familiar total de las familias con adulto mayor ubicadas en la Comuna Seis del municipio de Ibagué. Salud Uninorte. 2008;24:248-57.

42. Abdel Rahman TT, EI Gaafary MM. Elder mistreatment in a rural area in Egypt. Geriatr Gerontol Int. 2012;12:532-7. https://doi.org/10.1111/j.1447-0594.2011.00780.x

43. Dong X, Simon M, De Leon CM, Fulmer T, Beck T, Hebert $\mathbf{L}$, et al. Elder self-neglect and abuse and mortality risk in a community-dwelling population. JAMA. 2009;302:517-26. https://doi.org/10.1001/jama.2009.1109

44. Muñoz J. Personas mayores y malos tratos. Madrid: Psicología Pirámide; 2004. p. 10-40.

45. Organización Mundial de la Salud. Datos interesantes acerca del envejecimiento. Envejecimiento y ciclo de vida. Fecha de consulta: 3 de marzo de 2016. Disponible en: http://www.who.int/ageing/about/facts/es/

46. Instituto Nacional de Medicina Legal y Ciencias Forenses. Boletín trimestral. Violencia al adulto mayor en el contexto intrafamiliar. Fecha de consulta: 6 de abril de 2016. Disponible en: http://www.medicinalegal.gov.co/do cuments/10180/28521/3+BOLETINADULTOMAYOR.pdf/ a7c1d74e-d034-4e5d-a3ee-402d63148aa7

47. Cooper C, Selwood A, Livingston G. The prevalence of elder abuse and neglect: A systematic review. Age Ageing 2008;37:151-60. https://doi.org/10.1093/ageing/afm194.

48. Marmolejo I. Factores de riesgo del maltrato de personas mayores en la familia en población española. Rev Serv Soc. 2009;45:49-57.

49. Giraldo M. Análisis de la información estadística. Encuesta sobre maltrato a personas adultas mayores en el Distrito Federal 2006 (EMPAM-DF). Gobierno del Distrito Federal.
Fecha de consulta: 26 de marzo de 2016. Disponible en: http://www.geriatria.salud.gob.mx/descargas/investigacion/ analisis_EMPAMDF.pdf

50. Dolan V. Risk factors for elder abuse. J Insur Med 1999; 31:13-20.

51. Marks N. Gender issues in elder abuse. Journal of Marriage and Family. 1998;60:544-5.

52. Philips L. Domestic violence and aging women. Geriatr Nurs. 2000;21:188-93. https://doi.org/10.1067/mgn.2000.109584

53. Iborra I. Maltrato de personas mayores en la familia en España. Valencia: Fundación de la Comunitat Valenciana para el Estudio de la Violencia; 2008.

54. Sugiura Y, Tanimoto Y, Imbe A, Inaba Y, Sakai S, Shishikura $\mathrm{K}$, et al. Association between functional capacity decline and nutritional status based on the nutrition screening initiative checklist: A 2- year cohort study of Japanese CommunityDwelling Elderly. PloS One. 2016;11:e0166037. https://doi. org/10.1371/journal.pone.0166037

55. Dorantes G, Ávila JA, Mejía A, Gutiérrez LM. Factores asociados con la dependencia funcional en los adultos mayores: un análisis secundario del Estudio Nacional sobre Salud y Envejecimiento en México, 2001. Rev Panam Salud Pública. 2007;2:1-11. https://doi.org/10.1590/S102049892007000600001

56. Cortés C, Cardona D, Segura A, Garzón MO. Factores físicos y mentales asociados con la capacidad funcional del adulto mayor. Antioquia, Colombia, 2012. Rev Salud Pública (Bogotá). 2016;18:167-78. https://doi.org/10.15446/ rsap.v18n2.49237

57. Trize D, Conti MH, Gatti MA, Quintino NM, Simeão SF, Vitta A. Factors associated with functional capacity of elderly registered in the Family Health Strategy. Fisioter Pesqui. 2014;21:378-83. https://doi.org/10.590/1809-2950/ 13223421042014

58. Mai E, Tsubota M, Kikuya M, Satoh M, Inoue R, Hosaka $\mathbf{M}$, et al. Animal protein intake is associated with higher-level functional capacity in elderly adults: The Ohasama study. J Am Geriatr Soc. 2014;62:426-34. https://doi.org/10.1111/ jgs. 12690

59. Joweria N, Miho F, Tsukasa I. Nutritional status and functional capacity of community-dwelling elderly in southern Laos. Environ Health Prev Med. 2014;19:143-50. https://doi.org/10.1007/s12199-013-0367-1.

60. Organización Mundial de la Salud. La actividad física en los adultos mayores. Fecha de consulta: 6 de abril de 2016. Disponible en: http://www.who.int/dietphysicalactivity/ factsheet_olderadults/es/

61. Menéndez J, Guevara A, Arcia N, Díaz EM, Marín C, Alfonso JC. Enfermedades crónicas y limitación funcional en adultos mayores. Estudio comparativo en siete ciudades de América Latina y el Caribe. 2014. Fecha de consulta: 5 de abril de 2016. Disponible en: http://www.repositoriocdpd. net:8080/handle/123456789/371. 